\title{
Ozonização em meio básico para redução de cor do licor negro de indústria de celulose de algodão
}

\section{Color reduction of black liquor from cotton cellulose industry using ozonation in an alkaline medium}

\author{
José Roberto Guimarães \\ Professor livre-docente (associado) da Faculdade de Engenharia Civil, Arquitetura e Urbanismo da Universidade Estadual de Campinas (UNICAMP)
}

Ronaldo Leite Almeida Junior

Mestre e Doutorando da UNICAMP na área de Saneamento e Ambiente

\section{Milena Guedes Maniero}

Engenheira química formada pela Universidade Federal de São Carlos (UFSCar). Mestre em Engenharia Química pela UFSCar. Doutora em Engenharia Química na área de Tratamento de Águas pela Universidade Federal do Rio de Janeiro (UFRJ); pesquisadora da UNICAMP na área de Saneamento e Ambiente

\section{Pedro Sérgio Fadini}

Professor adjunto do Departamento de Química da UFSCar

\section{Resumo}

As indústrias de papel e celulose descartam no ambiente um grande volume de efluente contendo grande quantidade da substância lignina, que atribui coloração e apresenta considerável potencial de toxicidade. Neste trabalho, foi avaliada a ozonização em meio básico para a redução de cor do licor negro gerado por uma indústria de celulose de algodão. Face aos resultados, foi possível observar que, para menores concentrações iniciais de ozônio $\left(0,4\right.$ gO $\left.{ }_{3} \mathrm{~L}^{-1} \mathrm{~h}^{-1}\right)$, foi necessário um tempo mais longo de ozonização para se obter a redução desejada de 80\% da cor. O consumo específico de ozônio, entretanto, em comparação a experimentos com dosagens mais elevadas $\left(4,3 \mathrm{gO}_{3} \mathrm{~L}^{-1} \mathrm{~h}^{-1}\right)$ foi menor. Sugere-se que o oxigênio molecular desempenhe, também, um importante papel na oxidação dos compostos, participando do mecanismo de oxidação iniciado por radical hidroxila, ${ }^{\circ} \mathrm{OH}$, formado na ozonização em meio básico.

Palavras-chave: ozonização; POA; licor negro; redução de cor.

\section{Abstract}

Pulp and paper mills discharge large amounts of wastewater containing high concentrations of lignin, a coloring substance that is dangerous and presents high toxicity to the environment. In this study, ozonation in alkaline ambience was evaluated for color reduction in black liquor, generated in a cotton linter mill. It was observed that the ozonation time to reach $80 \%$ color reduction was higher at a lower initial ozone dose $\left(0,4 \mathrm{gO}_{3} \mathrm{~L}^{-1} \mathrm{~h}^{-1}\right)$ in comparison to a higher initial ozone dose $\left(4,3 \mathrm{gO}_{3} \mathrm{~L}^{-1} \mathrm{~h}^{-1}\right)$. On the other hand, the amount of consumed oxidant was lower at the lower ozone dose. It is suggested that molecular oxygen participates in the oxidation mechanism of colored compounds, which is initiated by hydroxyl radicals $\left({ }^{\circ} \mathrm{OH}\right)$ formed during ozonation in alkaline ambience.

Keywords: ozonation; AOP; black liquor; color.

\section{Introdução}

As indústrias de celulose e papel utilizam em seus processos grandes quantidades de água e, consequentemente, geram grandes volumes de efluentes líquidos. Essas águas residuárias possuem altos teores de sólidos em suspensão, matéria orgânica dissolvida, compostos organoclorados e, sobretudo, compostos coloridos, que possuem considerável potencial poluidor.

O licor negro é um dos principais subprodutos da indústria de papel. Esta corrente residual de processo apresenta elevada coloração devido à presença de grandes quantidades de compostos derivados de lignina. Podem conter cerca de 10 a 50\% de lignina em massa (ZAIED; BELLAKHAL, 2008; KSIBI et al, 2003).

A lignina é uma macromolécula aromática de estrutura química indefinida. A cor apresentada por suas soluções se deve à presença de moléculas com ligações duplas conjugadas com anéis aromáticos e grupos quinona e quinona metídio.

A concentração de lignina no efluente hídrico final da maioria das fábricas modernas de celulose não é mais tão elevada, uma vez que, para aproveitar o seu poder calorífico, esta corrente vem sendo 
concentrada e queimada para gerar energia. Porém, ainda existem empresas que não utilizam o processo de concentração e queima por razões técnicas e/ou econômicas.

O tratamento de efluentes aquosos industriais com base em processos biológicos é o mais utilizado devido às características de baixo custo e à possibilidade de tratamento de grandes volumes. Entretanto, a capacidade de certos micro-organismos de degradar determinados compostos orgânicos é limitada e os tratamentos biológicos convencionais são comprovadamente pouco eficientes na redução de cor de efluentes contendo contaminantes orgânicos coloridos (BERTAZZOLI; PELEGRINI, 2002).

A lignina e seus derivados são resistentes à biodegradação, principalmente devido à presença de ligações carbono-carbono do tipo bifenila (BAJPAI; MEHNA; BAJPAI, 1993). As estruturas da lignina têm a tendência de autocondensação, em especial em meio ácido, o que explica a sua resistência à degradação, que levaria à formação de estruturas moleculares mais simples (SAROJ et al, 2005). A presença de derivados da lignina no efluente resulta em grande aumento na demanda química de oxigênio (DQO) e na demanda bioquímica de oxigênio (DBO) (ZAIED; BELLAKHAL, 2009; KSIBI et al, 2003).

Apesar destas características, praticamente todas as indústrias de papel e celulose ainda adotam processos convencionais de tratamento dos efluentes, ou seja, sistemas baseados em processos biológicos com lodo ativado, lagoas aeradas, além de processos anaeróbios. Portanto, devido à remoção incompleta nas estações de tratamento de efluentes industriais, esses contaminantes podem estar sendo descartados em corpos d'água, poluindo águas e solos.

Os processos de remoção de cor mais comumente utilizados são os físico-químicos, nos quais se utilizam polímeros e outros insumos químicos, seguidos de coagulação, floculação, sedimentação ou flotação. Estes processos têm como desvantagem a geração de grande quantidade de resíduos sólidos. Atualmente, há sérias restrições ao licenciamento de mais aterros para a disposição dessa classe de material.

Diante disso, uma alternativa para a remoção de cor que evita a geração de lodos é a oxidação dos compostos coloridos por meio de processos oxidativos tradicionais, como adição de cloro, ozônio e dióxido de cloro, bem como por meio de processos oxidativos avançados (POA), como reação Fenton $\left(\mathrm{Fe}^{2+} / \mathrm{H}_{2} \mathrm{O}_{2}\right), \mathrm{UV} / \mathrm{H}_{2} \mathrm{O}_{2}, \mathrm{UV} / \mathrm{O}_{3}$, ozonização e outras técnicas e combinações de tecnologias oxidativas e microbiológicas (KREETACHAT et al, 2007; SAROJ et al, 2005; BELTRAN; GONZALEZ; GONZALEZ, 1997). Os processos oxidativos avançados envolvem a geração de radicais hidroxila $(\bullet \mathrm{OH})$, fortes agentes oxidativos.

Nakamura, Daidai e Kobayashi (2004), Bijan e Mohseni (2004) e Mantzavinos e Psillakis (2004) avaliaram o tratamento de efluentes industriais contendo lignina por ozonização e outros processos oxidativos. Ksibi et al (2003) mostraram que a fotocatálise heterogênea com o semicondutor $\mathrm{TiO}_{2}$, na presença de luz ultravioleta $\left(\mathrm{UV} / \mathrm{TiO}_{2}\right)$, foi capaz de aumentar a biodegradabilidade do licor negro. Torrades et al (2003) verificaram que o reagente Fenton, na presença de radiação solar UV $\left(\mathrm{Fe}^{2+} / \mathrm{H}_{2} \mathrm{O}_{2} / \mathrm{UV}\right)$, foi efetivo no tratamento de efluente da indústria de branqueamento de papel.

Apesar de seu potencial na degradação de efluentes da indústria papeleira, os POA apresentam como principal limitação o alto custo tanto de operação quanto de custo dos reagentes utilizados. Nesse contexto, a ozonização como uma primeira etapa de tratamento seguida de processos biológicos tem sido estudada no tratamento de diversos efluentes, incluindo os gerados pela indústria de celulose e papel. Um fator favorável é o fato de a molécula de lignina ser suscetível ao ataque de agentes eletrofílicos, como o ozônio (LANGLAIS; RECKHOW; BRINK, 1991).

O principal objetivo do presente trabalho foi investigar a aplicação de processo oxidativo avançado na redução de cor do licor negro gerado durante o processo de lavagem alcalina da celulose de línter. Devido às características do efluente em questão, o qual apresenta valores elevados de $\mathrm{pH}$, alcalinidade e vazão, avaliou-se experimentalmente o processo de ozonização em meio básico $\left(\mathrm{O}_{3} / \mathrm{OH}^{-}\right)$.

\section{Metodologia}

\section{Efluente}

As amostras de efluente foram coletadas na indústria Vicunha Têxtil, localizada na cidade de Americana, São Paulo. Foram coletadas no ponto denominado "tanque de espera de licor negro", que é o tanque que recebe o licor logo após ser gerado na lavagem alcalina.

Para a produção de celulose, o línter é submetido a banho alcalino, com adição de vapor, sob pressão. A lignina e outros compostos, como o óleo de algodão, são extraídos durante esse processo.

O licor negro obtido desse banho apresenta valores de $\mathrm{pH}$ variando entre 10 e 12, com forte alcalinidade; possui alta concentração de matéria orgânica, com DQO de aproximadamente $10.000 \mathrm{mg} \mathrm{L}^{-1}$, e uma intensa coloração negra, com valores próximos a $40.000 \mathrm{mg}$ Pt-Co L ${ }^{-1}$. A amostra não necessita de conservação por ser pouco biodegradável em virtude de seu caráter básico; além disso, os ensaios foram realizados imediatamente após a coleta.

Essa fábrica de celulose de línter emprega o processo de lodo ativado, aplicando oxigênio puro na oxidação do licor negro.

\section{Sistema experimental}

O sistema em que foram feitos os experimentos, mostrado na Figura 1, é constituído por reator de contato de 2 L (82,8 mm de diâmetro interno e altura útil de $230 \mathrm{~mm}$ ), com elemento poroso difusor de $2,6 \times 10^{5}$ poros $\mathrm{m}^{-2}$ e superfície útil de $0,0125 \mathrm{~m}^{2}$; frasco coletor de espumas (2 L) e frascos de trapeamento e monitoramento de ozônio, ambos de 2 L de capacidade.

$\mathrm{O}$ difusor poroso, operando com vazão de mistura gasosa $\mathrm{O}_{2}-\mathrm{O}_{3}$ de $1 \mathrm{~L} \mathrm{~min}^{-1}$, produz bolhas de diâmetro médio de 0,8 mm, medido 
por análise de imagens. Verificou-se, aqui, uma tendência à coalescência das bolhas na parte inferior do difusor.

O ozônio foi produzido com auxílio de gerador de descarga corona com capacidade nominal de $7 \mathrm{~g} \mathrm{O}_{3} \mathrm{~h}^{-1}$. A dosagem pode ser regulada por meio da tensão aplicada ao gerador de ozônio. O gás de entrada foi enriquecido em oxigênio por meio de sistema de peneiras moleculares integrantes do próprio aparelho (Marca Eaglesat, modelo OZOX 400/7).

\section{Procedimento experimental}

Os ensaios foram realizados em batelada. Depois da adição de $1 \mathrm{~L}$ do efluente a ser ensaiado no reator de contato, bem como de $200 \mathrm{~mL}$ da solução de iodeto de potássio ( $\left.20 \mathrm{~g} \mathrm{~L}^{-1}\right)$ em cada trap de absorção/ monitoramento, procedeu-se à ozonização por períodos de tempo determinados a uma vazão de gás de $1 \mathrm{~L} \mathrm{~min}^{-1}$.

O balanço de ozônio foi feito por meio de titulação com tiossulfato de sódio (APHA/AWWA/WEF, 2005). Os ensaios foram realizados com amostras originais em condições básicas: pH inicial entre 10 e 12 .

\section{Determinações analíticas}

A eficiência de remoção da coloração do efluente por meio de ozonização foi avaliada antes e após o tratamento, com auxílio de dois parâmetros físico-químicos: cor aparente e DQO. A demanda química de oxigênio foi determinada pelo método colorimétrico de refluxo fechado, segundo o Standard methods for the examination of water and wastewater (APHA/AWWA/WEF, 2005). As amostras brutas foram digeridas com 1,5 mL de $\mathrm{K}_{2} \mathrm{Cr}_{2} \mathrm{O}_{7}$ e 3,5 mL de $\mathrm{H}_{2} \mathrm{SO}_{4}$ a $150^{\circ} \mathrm{C}$ durante duas horas, e a absorbância, ao final, foi medida em espectrofotômetro DR4000U, em $600 \mathrm{~nm}$. A cor aparente foi determinada com padrões platina-cobalto, utilizando-se espectrofotômetro Odyssey DR2500, em $455 \mathrm{~nm}$.

\section{Modelos cinéticos}

Os dados cinéticos experimentais foram avaliados pelo método integral de análise de dados, conforme Levenspiel (2000). A ordem de uma reação é dada por uma das Equações 1 a 3, abaixo. Os experimentos para ajuste de modelos cinéticos foram feitos em triplicata.

$\mathrm{C}_{\mathrm{A}}=\mathrm{C}_{\mathrm{A} 0}-\mathrm{k} \cdot \mathrm{t}$

$\ln \left(\mathrm{C}_{\mathrm{A}} / \mathrm{C}_{\mathrm{AO}}\right)=\mathrm{k} \cdot \mathrm{t}$

$1 / C_{A}=1 / C_{A 0}+k \cdot t$

Onde:

$\mathrm{C}_{\mathrm{A} \text { : }}$ concentração do reagente no tempo t;

$\mathrm{C}_{\mathrm{A0} \text { : }}$ concentração inicial do reagente;

t: tempo;

k: coeficiente de velocidade de reação

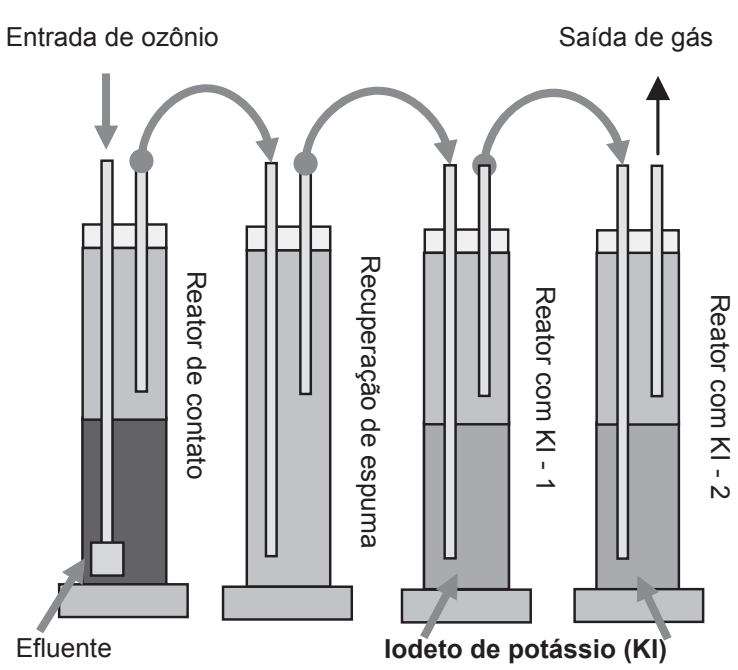

Figura 1 - Sistema de execução dos experimentos

\section{Resultados e discussão}

No processo de ozonização, no mínimo dois oxidantes podem atuar: o próprio ozônio, na forma molecular, e o radical hidroxila • OH. A reação direta ocorre em pH ácido, favorecendo a oxidação via ozônio molecular, que é mais seletivo. $\mathrm{O}$ aumento do pH ou a adição

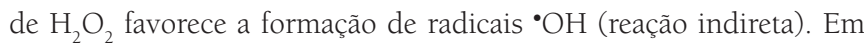
pH 7, ambos os oxidantes podem atuar. Na forma direta, a oxidação ocorre em meio ácido por adição eletrofílica do ozônio às cadeias insaturadas de alcenos e compostos aromáticos.

De acordo com a literatura, a ozonização é eficiente na oxidação de compostos que apresentam grupos aminas, anéis aromáticos e duplas ligações (Von GUNTEN, 2003).

\section{Redução da cor em função do tempo de ozonização/ozônio consumido}

Na Figura 2 são apresentados os resultados de remoção de cor em função do tempo de ozonização. Por meio de variação da dosagem específica inicial de ozônio $\left(0,4\right.$ a 4,3 $\left.\mathrm{g} \mathrm{O}_{3} \mathrm{~L}^{-1} \mathrm{~h}^{-1}\right)$, observou-se que foram necessários diferentes tempos para se atingir a remoção desejada de $80 \%$. Mesmo quando foi utilizada uma pequena dosagem inicial de ozônio $\left(0,4 \mathrm{~g} \mathrm{O}_{3} \mathrm{~L}^{-1} \mathrm{~h}^{-1}\right)$, foi possível obter uma apreciável remoção de cor com períodos de tempo de ozonização mais longos.

Como houve significativa redução da cor do efluente pela ozonização em meio básico, certamente, além da degradação dos grupos cromóforos, devem ter acontecido modificações estruturais nas moléculas dos componentes do efluente, aumentando a biodegradabilidade. Medeiros, Pires e Mohseni (2008a e 2008b), utilizando ozonização, comprovaram a redução de cor, a remoção de compostos orgânicos de alta massa molar e o aumento da biodegradabilidade de efluentes de uma planta de lixívia alcalina. 


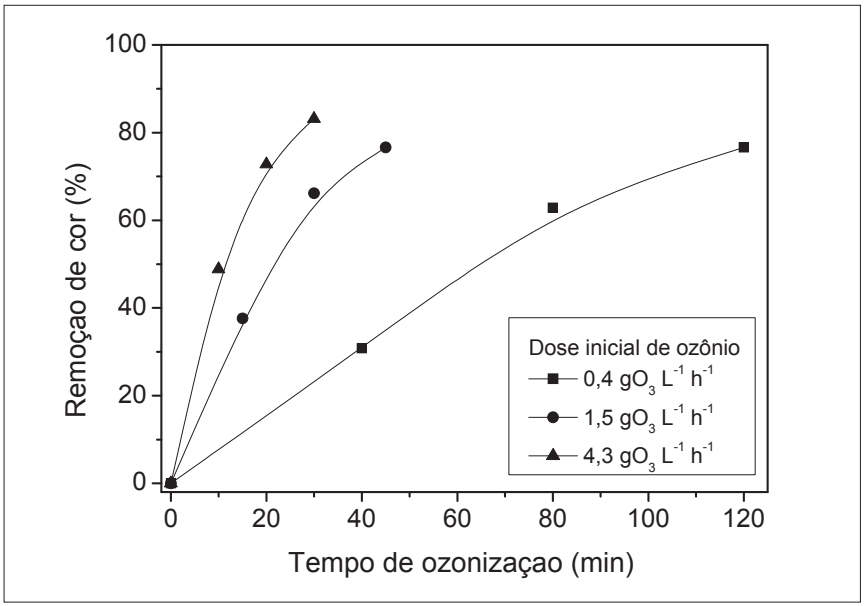

Figura 2 - Remoção de cor em função do tempo de ozonização para diferentes dosagens iniciais de ozônio

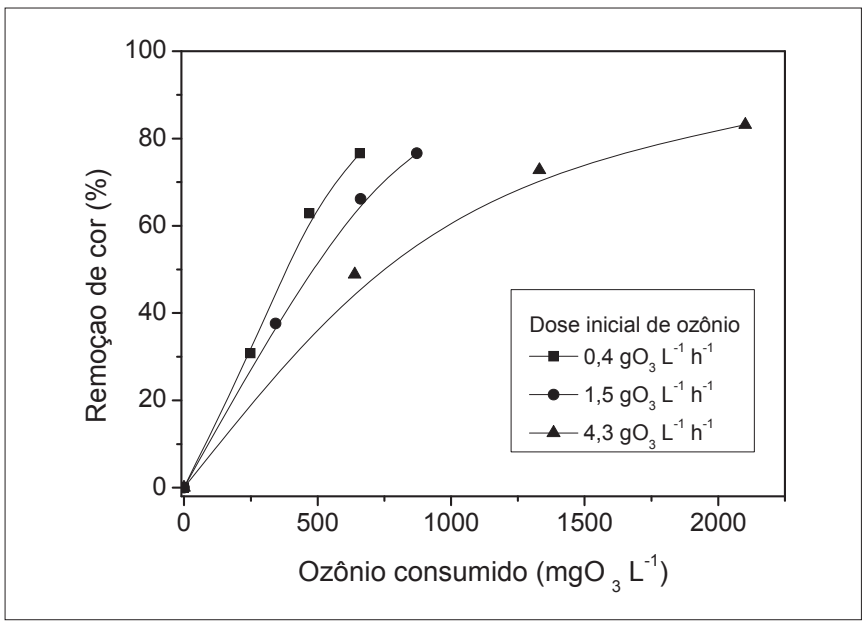

Figura 3 - Remoção de cor em função do consumo de ozônio para diferentes dosagens iniciais de ozônio

Na Figura 3 é apresentada a eficiência de remoção de cor em função do consumo de ozônio para dosagens iniciais de ozônio que variem de 0,4 a 4,3 $\mathrm{g} \mathrm{O}_{3} \mathrm{~L}^{-1} \mathrm{~h}^{-1}$ Durante a ozonização, é de fundamental importância a avaliação do consumo de ozônio, uma vez que a concentração de ozônio consumido determina a viabilidade econômica do processo, caso seja viável do ponto de vista técnico.

Analisando-se a Figura 2, é possível constatar que, quando se utiliza menor concentração inicial de ozônio $\left(0,4 \mathrm{~g} \mathrm{O}_{3} \mathrm{~L}^{-1} \mathrm{~h}^{-1}\right)$, é necessário um maior tempo de ozonização para se obter a remoção desejada de $80 \%$. Entretanto, observa-se que, apesar do tempo de ozonização mais prolongado, necessário para a oxidação, o consumo de ozônio foi inferior, conforme Figura 3.

O menor consumo de ozônio, utilizando-se menor dosagem inicial de ozônio, pode ser explicado pela reação entre o oxigênio molecular e alguns radicais orgânicos suscetíveis ao ataque do oxigênio dissolvido em concentração mais alta. Durante a ozonização em meio básico, o radical hidroxila combina-se com os compostos orgânicos, formando outros radicais orgânicos que podem ser suscetíveis ao ataque pelo oxigênio. Dessa forma, há um consumo menor de ozônio durante o processo de oxidação (PAN; SCHUCHMANN; VON SONNTAG et al, 2000).

\section{Redução da DQO durante a ozonização}

A remoção de DQO durante a ozonização variou de 0 a 20\%. Essa relativa baixa remoção se deve ao elevado valor de DQO do efluente avaliado (mais de $10.000 \mathrm{mg} \mathrm{L}^{-1}$ ) e, provavelmente, às dosagens de ozônio aplicadas (que não provocaram redução perceptível da matéria orgânica), uma vez que, conforme Mansson e Oster (1998), o ozônio reage com compostos providos de anéis aromáticos presentes na lignina, com consequente formação de derivados de ácido mucônico e outros compostos contendo grupos carboxila, causando pequena variação na matéria orgânica passível de oxidação.

\section{Cinética de oxidação de compostos coloridos}

Segundo Langlais, Reckhow e Brink (1991), na maioria dos casos, a taxa de oxidação de compostos coloridos pode ser mais bem interpretada e apresentada como uma reação de pseudo-primeira ordem. Pelos resultados obtidos neste trabalho, confirma-se o modelo cinético de velocidade de reação de pseudo-primeira ordem, conforme Equação 4.

$\ln \left([\mathrm{C}] /\left[\mathrm{C}_{0}\right]\right)=\mathrm{k}_{\mathrm{tc}} \cdot \mathrm{t}$

Equação 4

Onde:

[C]: cor da solução (mg Pt-Co L-1);

$\left[\mathrm{C}_{0}\right]$ : cor inicial da solução (mg Pt-Co L-1);

$\mathrm{k}_{\mathrm{tc}}$ : é o coeficiente cinético $\left(\mathrm{min}^{-1}\right)$, que depende das dosagens iniciais de ozônio;

t: é o período de tempo de oxidação relativo à [C], em minutos.

\section{Determinação do coeficiente cinético de reação $\left(k_{t c}\right)$ em função da dosagem inicial de ozônio}

Conforme apresentado na Figura 2, verificou-se que existe uma diferença de desempenho da ozonização em função da dosagem inicial de ozônio. Com o objetivo de avaliar a relação entre a cinética de reação e a dosagem inicial de ozônio, foram obtidos os coeficientes cinéticos das reações de oxidação de compostos coloridos $\left(\mathrm{k}_{\mathrm{tc}}\right)$ em função das dosagens iniciais de ozônio. Na Equação 5 é apresentada a correlação entre esses parâmetros.

$\mathrm{k}_{\mathrm{tc}}=-0,0123 \ln ($ dose $)-0,0199$

Equação 5

Onde:

$\mathrm{k}_{\mathrm{tc}}$ : coeficiente cinético de oxidação $\left(\mathrm{min}^{-1}\right)$;

dose: dosagem inicial de ozônio $\left(\mathrm{mg} \mathrm{O}_{3} \mathrm{~L}^{-1} \mathrm{~h}^{-1}\right)$. 
Como já se sabe, ao observar a Equação 5 verifica-se a dependência de ordem logarítmica entre o coeficiente cinético e a dosagem inicial de ozônio, fato de extrema relevância aqui e para o caso de dimensionamento de um sistema real.

\section{Determinação do coeficiente cinético de consumo de ozônio $\left(k_{d}\right)$ em função da dosagem inicial de ozônio}

Conforme apresentado na Figura 3, verifica-se a diferença de desempenho da remoção de cor em função do consumo de ozônio quando empregadas diferentes dosagens iniciais de ozônio. Com o objetivo de avaliar essa relação, foi obtido o coeficiente cinético de consumo de ozônio das reações de oxidação de compostos coloridos $\left(k_{d}\right)$ em função da dosagem inicial de ozônio. As equações de reação de primeira ordem foram adequadas aos dados experimentais, e os coeficientes foram obtidos por ajuste de retas em gráfico monolog, pelo método dos mínimos quadrados. Na Equação 6 é apresentada essa correlação:

$\mathrm{k}_{\mathrm{d}}=0,0003 \ln ($ dose $)-0,0016$

Equação 6

Na qual:

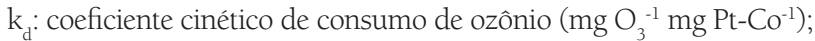
dose: dosagem específica inicial de ozônio $\left(\mathrm{mg} \mathrm{O}_{3} \mathrm{~L}^{-1} \mathrm{~h}^{-1}\right)$.

Mais uma vez, verifica-se que há dependência de ordem logarítmica entre o coeficiente cinético de consumo de ozônio e a dosagem inicial de ozônio, fato que deve ser observado no projeto de um sistema de ozonização.

\section{Participação do oxigênio molecular na ozonização}

Segundo Pan, Schuchmann e von Sonntag (2000), os radicais hidroxila atacam regiões moleculares de alta densidade eletrônica, adicionando-se às ligações insaturadas de compostos aromáticos e alcenos. $\mathrm{O}$ ataque de radicais hidroxila a anéis benzênicos resulta na formação de radicais hidrociclohexadienila, ao passo que o ataque de oxigênio a estes últimos proporciona a formação de endoperoxialquila e radicais endoperoxila; os radicais endoperoxila podem formar, então, endoperóxidos.

Os radicais hidroxila podem também ser responsáveis pela abstração de átomos de hidrogênio em cadeia saturada de hidrocarbonetos, resultando em sítios radicalares. Esses sítios podem ser atacados pelo oxigênio, acarretando a formação de cadeias insaturadas e radicais hidroperoxila.

A participação do oxigênio molecular em POA ocorre na medida em que um composto orgânico é atacado pelo radical hidroxila e forma outros radicais orgânicos suscetíveis ao ataque pelo oxigênio.

Como há a participação do oxigênio na reação, é possível que, em processo que utiliza ozônio como oxidante de forma indireta
Tabela 1 - Coeficientes cinéticos calculados em função da dosagem específica de ozônio

\begin{tabular}{lcc} 
Dosagem $\left(\mathrm{O}_{3} \mathrm{~L}^{-1} \mathrm{~h}^{-1}\right)$ & $\mathrm{k}_{\mathrm{tc}}\left(\mathrm{min}^{-1}\right)$ & $\mathrm{k}_{\mathrm{d}}\left(\mathrm{mg} \mathrm{O}_{3}^{-1}{\left.\mathrm{mg} \mathrm{Pt}-\mathrm{Co}^{-1}\right)}^{-0,0086}\right.$ \\
0,4 & $-0,038$ & $-0,0019$ \\
4,3 & 0,0012 \\
$\begin{array}{l}\text { Relação entre dosagens } \\
(\mathrm{D}=0,4 / \mathrm{D}=4,3)\end{array}$ & 0,23 & 1,6 \\
\hline
\end{tabular}

(em meio básico), ao se controlar a dosagem inicial de ozônio, os radicais hidroxila formados sejam responsáveis pelo início do ataque às moléculas orgânicas e, posteriormente, ocorra o ataque pelo oxigênio, conforme os mecanismos já comentados. Deve-se salientar a necessidade do controle da dosagem inicial de ozônio para que seja formada a quantidade de radicais hidroxila necessária para dar início à oxidação.

No presente trabalho isso deve ter acontecido. O resultado perceptível foi uma redução da massa de ozônio utilizada na reação, porém com o prejuízo do aumento do período de tempo de reação. Portanto, pelos resultados obtidos, foi possível observar que, além de a concentração de ozônio no gás oxidante constituir um importante parâmetro de controle do sistema, não menos importante é a presença do oxigênio dissolvido no reator de contato, uma vez que este participa das reações de oxidação dos intermediários formados pelos radicais hidroxila.

A possibilidade de aplicação de uma técnica para diminuir o consumo de ozônio com a utilização de oxigênio parece vantajosa porque, a grosso modo, para a geração de 1 kg de ozônio são necessários $10 \mathrm{~kg}$ de oxigênio a 99,5\% de pureza e mais $10 \mathrm{kWh}$ para o processo corona, enquanto para a produção de $1 \mathrm{~kg}$ de oxigênio é consumido 0,4 kWh em plantas criogênicas com média de idade de 25 anos (LANGLAIS et al, 1991).

\section{Comparação do consumo de ozônio para a remoção de cor}

As Equações 5 e 6 foram utilizadas para os cálculos dos coeficientes cinéticos em função da dosagem específica de ozônio $(0,4$ e 4,3 g $\left.\mathrm{O}_{3} \mathrm{~L}^{-1} \mathrm{~h}^{-1}\right)$, resultados apresentados na Tabela 1 .

Analisando-se os dados da Tabela 1 e comparando-se as dosagens específicas de 0,4 e 4,3 $\mathrm{g} \mathrm{O}_{3} \mathrm{~L}^{-1} \mathrm{~h}^{-1}$, verifica-se que, utilizando a menor dosagem $\left(0,4 \mathrm{~g} \mathrm{O}_{3} \mathrm{~L}^{-1} \mathrm{~h}^{-1}\right)$, é necessário um tempo de reação 4,3 vezes maior. Porém, o consumo de ozônio é 1,6 vezes menor, para a obtenção a mesma redução de cor do efluente desejada (80\%), quando se utiliza ozonização em meio básico.

\section{Conclusões}

Neste trabalho, o cálculo do consumo de ozônio, bem como de suas relações, permitiram inferir conclusões que podem ser utilizadas na aplicação de ozonização e suas combinações para tratamento oxidativo de efluentes 
A redução de cor do licor negro por meio de processo de ozonização admite ser tratada como uma reação de primeira ordem.

Os resultados obtidos sugerem que o oxigênio molecular tem grande importância na oxidação dos compostos coloridos do efluente. Diversas espécies químicas são capazes de reagir com o radical hidroxila, gerando outros radicais orgânicos que, por sua vez, reagem mais facilmente com o oxigênio molecular. Assim, conclui-se que o oxigênio é capaz de complementar a oxidação inicial promovida pelos radicais $\bullet \mathrm{OH}$ formados durante a ozonização em pH básico. Tal resultado é de grande importância, uma vez que o consumo de ozônio pode ser minimizado pelo aumento do tamanho do reator e, consequentemente, do tempo de residência.

\section{Agradecimentos}

Os autores agradecem à Coordenação de Aperfeiçoamento de Pessoal de Nível Superior (Capes), ao Conselho Nacional de Desenvolvimento Científico e Tecnológico (CNPq) e à Fundação de Amparo à Pesquisa do Estado de São Paulo (FAPESP) pelo apoio financeiro.

\section{Referências}

APHA/AWWA/WEF. Standard methods for the examination of water and wastewater. $21^{\text {th }}$ ed. American Public Health Association: Washington, DC, 2005

BAJPAI, P.; MEHNA, A.; BAJPAI, P.K. Decolorization of kraft bleach plant effluent with the white-rot fungus trametes versicolor, Process Biochemistry, v. 28, n. 6, p. 377-384, 1993.

BELTRAN, F.J.; GONZALEZ, M.; GONZALEZ, J.F. Industrial wastewater advanced oxidation. part-i: uv radiation in presence and absence of hydrogen peroxide. Water Research, v. 31, n. 10, p. 2405-2414, 1997.

BERTAZZOLI, R.; PELEGRINI, R. Descoloração e degradação de poluentes orgânicos em soluções aquosas através do processo fotoeletroquímico. Química Nova, v. 25, n. 3, p. 477-482, 2002.

BIJAN, L.; MOHSENI, M. Using ozone to reduce recalcitrant compounds and to enhance biodegradability of pulp and paper effluents. Water Science and Technology, v. 50, n. 3, p. 173-182, 2004.

KREETACHAT, T. et al. Effects of ozonation process on lignin-derived compounds in pulp and paper mill effluents, Journal of Hazardous Materials, v. 142, n. 1-2, p. 250-257, 2007.

$\mathrm{KSIBI}, \mathrm{M}$. et al. Photodegradation of lignin from black liquor using a UV/ $\mathrm{TiO}_{2}$ system. Journal of Photochemistry \& Photobiology A: Chemistry, v. 154, n. 2-3, p. 211-218, 2003

LANGLAIS, B.; RECKHOW, D.A.; BRINK, D.R. Ozone in water treatment: application and engineering. Chelsea: AWWA Research Foundation, Lewis Publishers, 1991.

LEVENSPIEL, O. Engenharia das reações químicas. São Paulo, Editora Edgard Blücher Ltda, 2000

MÀNSSON, P.; OSTER, R. Ozonization of kraft lignin. Nordic Pulp and Paper Research Journal, v. 3, n. 2, p. 75-81, 1988.
MANTZAVINOS, D.; PSILLAKIS, E. Enhancement of biodegradability of industrial wastewaters by chemical oxidation pre-treatment. Journal of Chemical Technology and Biotechnology, v. 79, n. 5, p. 431-454, 2004.

MEDEIROS, D.R.; PIRES, E.C.; MOHSENI, M. Ozone oxidation of pulp and paper wastewater and its impact on molecular weight distribution of organic matter. Ozone Science \& Engineering, v. 30, n. 1, p. 105-110, 2008a.

Ozonização do efluente de extração alcalina e seu impacto sobre a biodegradabilidade e distribuição do peso molecular dos compostos orgânicos. O Papel, v. 3, p. 49-59, 2008b.

NAKAMURA, Y.; DAIDAI, M.; KOBAYASHI, F. Ozonolysis mechanism of lignin model compounds and microbial treatment of organic acids produced. Water Science and Technology, v. 50, n. 3, p. 167-172, 2004.

PAN, X.M.; SCHUCHMANN, M.N.; VON SONNTAG, C. Oxidation of benzene by the $\mathrm{OH}$ radical. A product and pulse radiolysis study in oxygenated aqueous solution. Journal of the Chemical Society, Perkin Transactions 2, n. 3, p. 289-297, 1993.

SAROJ, D.P. et al. Mineralization of Some Natural Refractory Organic Compounds by Biodegradation and Ozonation, Water Research, v. 39 n. 9, p. 1921-1933, 2005

TORRADES, F. et al. Experimental design of fenton and photo-fenton reactions for the treatment of cellulose bleaching effluents, Chemosphere, v. 53, n. 10, p. $1211-1220,2003$

Von GUNTEN, U. Ozonation of drinking water: Part I. Oxidation kinetics and product formation. Water Research, v. 37, n. 7, p. 1443-1467, 2003.

ZAIED, M.; BELLAKHAL, N. Electrocoagulation treatment of black liquor from paper industry. Journal of Hazardous Materials, v. 163, n. 2-3, p. 995-1000, 2009. 